\title{
ON 'BEST' RATIONAL APPROXIMATIONS TO $\pi$ AND $\pi+e$
}

\author{
Bertrand Teguia Tabuguia
}

Kassel University, Heinrich-Plett-Str.40. 34132 Kassel

bertrand.teguia@aims-cameroon.org

\begin{abstract}
Through the half-unit circle area computation using the integration of the corresponding curve power series representation, we deduce a slow converging positive infinite series to $\pi$. However, by studying the remainder of that series we establish sufficiently close inequalities with equivalent lower and upper bound terms allowing us to estimate, more precisely, how the series approaches $\pi$. We use the obtained inequalities to compute up to four-digit denominator, what are in this sense, the best rational numbers that can replace $\pi$. It turns out that the well-known convergents of the continued fraction of $\pi, 22 / 7$ and 355/113 called, respectively, Yuelü and Milü in China are the only ones found. Thus we apply a similar process to find rational estimations to $\pi+e$ where $e$ is taken as the power series of the exponential function evaluated at 1 . For rational numbers with denominators less than 2000 , the convergent $920 / 157$ of the continued fraction of $\pi+e$ turns out to be the only rational number of this type.
\end{abstract}

Keywords: $\pi$ (read Pi); $e$ (the natural logarithm base); continued fraction; convergent infinite series.

\section{Introduction}

$\pi$, the ratio of a circle's circumference to its diameter, and $e$, the limit of $n \mapsto(1+1 / n)^{n}$ for a positive integer $n$, are two transcendental numbers, that is, they cannot be solutions of non-constant polynomial equations with integer coefficients. In particular, there is no linear equation over the field of rationals satisfied by these two numbers and this is equivalent to say that $\pi$ and $e$ are irrational numbers, which means, they cannot be written as ratios of two integers. However, it is still unknown whether $\pi+e$ is irrational or not. Speaking of irrationality one comes to the structure of these numbers and how they can be represented. The two most common ways to represent irrational numbers are the decimal and the continued fraction representations. 
Apart from numbers like the Champernowne constant $C_{10}=0.1234567891011 \ldots$ that are constructed from a decimal representation, the irrational numbers generally have digits that appear as incomprehensible in their decimal representations. One can cite $\sqrt{2}=1.414213562373095 \ldots$ as an example. This explains the endless character of the fascinating mathematicians and computer scientists 'sport' whose aim is to compute digits of the decimal representations of popular irrational numbers. The case of $\pi$ remains the most played and there are many great results related to its computations. For about three decades all the records are obtained using the Chudvnosky algorithm based on Ramanujan's $\pi$ formulas Agarwal et al. (2013). At the current date, the record belongs to Timothy Mullican who computed 50 trillion digits of $\pi$ in 10 months Mullican (2020). Another important result to mention is Bailey et al. (1997) which gives a method to compute the $n^{\text {th }}$ digit of $\pi$ without computing the preceding $n-1$ digits. Concerning $e$ the computations of digits are not that attractive. Good decimal approximations can easily be computed using the infinite series

$$
\sum_{n=0}^{\infty} \frac{1}{n !}=1+\frac{1}{1}+\frac{1}{2 \cdot 1}+\frac{1}{3 \cdot 2 \cdot 1}+\cdots
$$

which converges incredibly fast to $e$. This fact leads to a simple proof of the irrationality of $e$ funnily seen as an 'unnatural' convergence because the limit is too well approximated by rational numbers (terms of summation in (1)). Obviously, neither rationals obtained from a partial sum of an infinite series nor those from the rational representation of a decimal approximation which rather yield, more complicated, fractions in general are part of our interest. The result of this paper is more likely connected to the regular or canonical continued fraction representation that we briefly recall next.

The regular continued fraction of a real number $x$ is generally given as

$$
\lim _{n \rightarrow \infty}\left[c_{0} ; c_{1}, c_{2}, \ldots, c_{n-1}, c_{n}\right],
$$

where $c_{0}=\lfloor x\rfloor$ is the greatest integer less than $x, c_{1}=\left\lfloor 1 /\left(x-c_{0}\right)\right\rfloor, c_{2}=\lfloor 1 /(1 /(x-$ $\left.\left.\left.c_{0}\right)-c_{1}\right)\right\rfloor$, etc Pettofrezzo and Byrkit (1970). This process terminates for every rational number but it is infinite for each irrational number. We write

$$
x=c_{0}+\frac{1}{c_{1}+\frac{1}{c_{2}+\frac{1}{\ddots+\frac{\ddots}{c_{n-1}+\frac{1}{c_{n}+\ddots}}}} .}
$$

The use of continued fraction representations dates back to Euler to whom we owe a vast majority of known formulas related to $\pi$ Eymard and Lafon (1999). Unlike the decimal representation, the continued fractions of many popular irrational numbers present patterns in their representations. In particular, we have

$$
e=[2 ; 1,2,1,1,4,1,1,6,1,1,8, \ldots, 1,1,2 \cdot n, 1,1, \ldots]
$$


which was given by Euler to establish the first proof of the irrationality of $e$. In 1761, Euler's contemporary mathematician Johann Lambert gave the first proof of the irrationality of $\pi$. Lambert used a general continued fraction to show that the tangent function does not assume rational numbers, which implies in particular that $\pi$ is irrational since $\tan (\pi / 4)=1$ is rational Agarwal et al. (2013). He also gave the following starting of the regular continued fraction of $\pi$

$$
\pi=[3 ; 7,15,1,295,1,1,1,2, \ldots] .
$$

Unfortunately, no pattern has ever been found in the regular continued fraction of $\pi$. If this was not the case then the regular continued fraction of $\pi+e$ may have been approachable using the arithmetic of continued fractions developed in Gosper (2015).

Let us now come back to the definition in (2). We call a convergent of the continued fraction represented by (2) of an irrational number $x$, any rational number obtained with the finite continued fraction

$$
\left[c_{0} ; c_{1}, c_{2}, \ldots, c_{n}\right]=c_{0}+\frac{1}{c_{1}+\frac{1}{c_{2}+\frac{1}{\ddots \cdot \frac{1}{\frac{1}{c_{n}}}}}},
$$

where $n$ is a non-negative integer. Such a rational number generally provides a 'good' approximation to $x$. In the case of $\pi$, some convergents are

$$
\frac{3}{1}, \frac{22}{7}, \frac{333}{106}, \frac{355}{115}, \frac{103993}{33102} .
$$

The numbers $22 / 7$ and $355 / 115$ which were well known much earlier before the development of continued fractions present an important common particularity. In Beukers (2000) the author defined the quality of a rational approximation to $\pi$ as the number $m$ such that

$$
\left|\pi-\frac{p}{q}\right|=\frac{1}{q^{m}} .
$$

We have $m \approx 3.429$ for $22 / 7$ and $m \approx 3.201$ for $355 / 115$. The maximum value of $m \geqslant 2$ for which there are infinitely many pairs of integers $(p, q)$ with quality $m$ is called the irrationality measure 1 . For $\pi$ this value is known to be at most $7.103205334137 . .$. Zeilberger and Zudilin (2020). Nevertheless, up to four digitdenominator none rational approximations reach the qualities of $22 / 7$ or $355 / 113$. We are going to build algorithms 1 and 2 that extract such good rational approximations from the convergents of $\pi$ and $\pi+e$, respectively.

\footnotetext{
${ }^{1}$ Note that by definition of the irrational measure one uses $\leqslant$ instead of $=$ in $(6)$
} 


\section{Some properties}

The first elaborated approach to compute decimal of $\pi$ was proposed by Archimedes. The idea was to bound a circle of radius $r$ with polygons of many sides. Then the area $\pi \cdot r^{2} 2$ of the circle comes close to the polygons ones as its number of side increases. Nowadays with modern mathematics, this could be seen differently. In a coordinate system, let us consider the upper half unit circle curve equation $y=\sqrt{1-x^{2}},|x| \leqslant 1$. Using integration one computes the area under the curve as

$$
\frac{\pi}{2}=\int_{-1}^{1} \sqrt{1-x^{2}}
$$

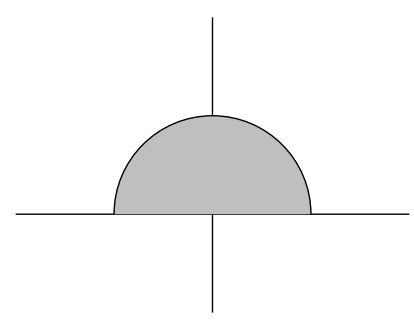

Figure 1: Half unit circle area $\frac{\pi}{2}=\int_{-1}^{1} \sqrt{1-x^{2}} d x$

On the other hand $x \mapsto \sqrt{1-x^{2}}$ is analytic over $(-1,1)$ and therefore can be expressed as a convergent power series. The corresponding power series is easily handled using formal computations. In Teguia T. (2020) this author developed a complete algorithm for computing power series representations of a very large family of expressions. The expression that we are considering here is much easier and we get

$$
\sqrt{1-x^{2}}=-\sum_{n=0}^{\infty} \frac{(2 \cdot n) ! \cdot x^{2 n}}{(2 \cdot n-1) \cdot 4^{n} \cdot n !^{2}}=-\sum_{n=0}^{\infty} \frac{\left(\begin{array}{c}
2 \cdot n \\
n
\end{array}\right) \cdot x^{2 n}}{(2 \cdot n-1) \cdot 4^{n}} .
$$

Since $\frac{\left(\begin{array}{c}2 \cdot n \\ n\end{array}\right) \cdot x^{2 n}}{(2 \cdot n-1) \cdot 4^{n}} \geqslant 0$ for all $x \in[-1,1]$ and integers $n \geqslant 1$, from $(7)$ and $(8)$ and the Fubini theorem it follows that

$$
\begin{aligned}
\pi & =-2 \int_{-1}^{1} \sum_{n=0}^{\infty} \frac{\left(\begin{array}{c}
2 \cdot n \\
n
\end{array}\right) \cdot x^{2 n}}{(2 \cdot n-1) \cdot 4^{n}} d x \\
& =-2 \sum_{n=0}^{\infty} \frac{\left(\begin{array}{c}
2 \cdot n \\
n
\end{array}\right)}{(2 \cdot n-1) \cdot 4^{n}} \int_{-1}^{1} x^{2 n} d x \\
& =-\sum_{n=0}^{\infty} \frac{\left(\begin{array}{c}
2 \cdot n \\
n
\end{array}\right)}{(2 \cdot n-1) \cdot(2 \cdot n+1) \cdot 4^{n-1}} .
\end{aligned}
$$

\footnotetext{
${ }^{2}$ Archimedes original idea was made for the circumference computations Beukers (2000).
} 
The latter can be even more refined. And it is in doing so that we arrive at the central theorem of this article.

Theorem 1. [Some formulas for $\pi$ ] The number $\pi$, the unit circle area satisfy

$$
\pi=\frac{1}{2} \sum_{n=0}^{\infty} \frac{\left(\begin{array}{c}
2 \cdot n \\
n
\end{array}\right)}{(2 \cdot n+1) \cdot 4^{n-1}}
$$

and moreover, for all positive integers $n$

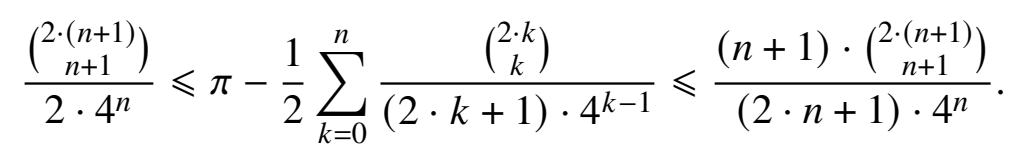

Proof. By partial fraction decomposition we have

$$
\frac{1}{(2 \cdot n-1) \cdot(2 \cdot n+1)}=\frac{1}{2 \cdot(2 \cdot n-1)}-\frac{1}{2 \cdot(2 \cdot n+1)} .
$$

Therefore from (9) the first formula of the theorem follows if and only if

$$
\sum_{n=0}^{\infty}-\frac{\left(\begin{array}{c}
2 \cdot n \\
n
\end{array}\right)}{2 \cdot(2 \cdot n-1) \cdot 4^{n-1}}=0
$$

We show this by applying Gosper's algorithm (see Gosper (1978)). Indeed, it turns out that the partial sum of the given infinite series is a hypergeometric term with a hypergeometric summand (we say that it is Gosper summable). One gets

$$
-\frac{\left(\begin{array}{c}
2 \cdot k \\
k
\end{array}\right)}{2 \cdot(2 \cdot k-1) \cdot 4^{k-1}}=s_{k+1}-s_{k}=\frac{(k+1) \cdot\left(\begin{array}{c}
2 \cdot(k+1) \\
k+1
\end{array}\right)}{(2 \cdot k+1) \cdot 4^{k}}-\frac{k \cdot\left(\begin{array}{c}
2 \cdot k \\
k
\end{array}\right)}{(2 \cdot k-1) \cdot 4^{k-1}},
$$

and therefore by telescoping we get

$$
\sum_{k=1}^{n}-\frac{\left(\begin{array}{c}
2 \cdot k \\
k
\end{array}\right)}{2 \cdot(2 \cdot k-1) \cdot 4^{k-1}}=s_{n+1}-s_{0}=\frac{(n+1) \cdot\left(\begin{array}{c}
2 \cdot(n+1) \\
n+1
\end{array}\right)}{(2 \cdot n+1) \cdot 4^{n}} .
$$

Hence

$$
\sum_{n=0}^{\infty}-\frac{\left(\begin{array}{c}
2 \cdot n \\
n
\end{array}\right)}{2 \cdot(2 \cdot n-1) \cdot 4^{n-1}}=\lim _{n \rightarrow \infty} \frac{(n+1) \cdot\left(\begin{array}{c}
2 \cdot(n+1) \\
n+1
\end{array}\right)}{(2 \cdot n+1) \cdot 4^{n}}=0 .
$$

The limit holds because for all positive integers $n$

$$
\begin{aligned}
\frac{\left(\begin{array}{c}
2 \cdot(n+1) \\
(n+1)
\end{array}\right)}{4^{n}} & =\frac{(2 \cdot n+2) \cdot(2 \cdot n+1) \cdot(2 \cdot n) \cdot(2 \cdot n-1) \cdots(n+2)}{4^{n} \cdot(n+1) \cdot n \cdot(n-1) \cdots 1} \\
& \leqslant\left\{\begin{array}{l}
\text { if } n+1 \text { is even : } \\
\frac{(2 \cdot n+2) \cdot(2 \cdot n+2) \cdot(2 \cdot n) \cdot(2 \cdot n) \cdots(n+3) \cdot(n+3)}{4^{n} \cdot(n+1) \cdot n \cdot(n-1) \cdots 1} \\
\text { if } n+1 \text { is odd : } \\
\frac{(2 \cdot n+2) \cdot(2 \cdot n+2) \cdot(2 \cdot n) \cdot(2 \cdot n) \cdots(n+4) \cdot(n+4) \cdot(n+2)}{4^{n} \cdot(n+1) \cdot n \cdot(n-1) \cdots 1},
\end{array}\right. \\
= & \frac{4^{\lfloor(n+1) / 2\rfloor} \cdot 2^{n \equiv 2}}{4^{n} \cdot\left\lfloor\frac{n+1}{2}\right\rfloor !}=\frac{2^{n \equiv 2}}{4^{\lceil n / 2\rceil}\left\lfloor\frac{n+1}{2}\right\rfloor !} \longrightarrow 0, \text { as } n \rightarrow 0 .
\end{aligned}
$$


$n \equiv 2$ denotes the remainder in the division by 2 , and $\lceil x\rceil$ the smallest integer greater than $x$. Note that this limit computation can also easily be deduced by means of the connection between the Wallis product and the central binomial coefficient $\left(\begin{array}{c}2 \cdot n \\ n\end{array}\right)$.

For the second part of the proof, we study the remainder of the infinite series on the right hand side of $(10)$. We have

$$
\pi-\frac{1}{2} \sum_{k=0}^{n} \frac{\left(\begin{array}{c}
2 \cdot k \\
k
\end{array}\right)}{(2 \cdot k+1) \cdot 4^{k-1}}=\frac{1}{2} \sum_{k=n+1}^{\infty} \frac{\left(\begin{array}{c}
2 \cdot k \\
k
\end{array}\right)}{(2 \cdot k+1) \cdot 4^{k-1}} .
$$

However for all positive integers $k$

$$
0<2 \cdot k-1<2 \cdot k+1<2 \cdot k+2 \Rightarrow \frac{1}{2 \cdot k+2}<\frac{1}{2 \cdot k+1}<\frac{1}{2 \cdot k-1} .
$$

Since $\frac{\left(\begin{array}{c}2 \cdot k \\ k\end{array}\right)}{4^{k-1}}>0$, after multiplication and summation we get

$$
\frac{1}{2} \sum_{k=n+1}^{\infty} \frac{\left(\begin{array}{c}
2 \cdot k \\
k
\end{array}\right)}{(2 \cdot k+2) \cdot 4^{k-1}} \leqslant \frac{1}{2} \sum_{k=n+1}^{\infty} \frac{\left(\begin{array}{c}
2 \cdot k \\
k
\end{array}\right)}{(2 \cdot k+1) \cdot 4^{k-1}} \leqslant \frac{1}{2} \sum_{k=n+1}^{\infty} \frac{\left(\begin{array}{c}
2 \cdot k \\
k
\end{array}\right)}{(2 \cdot k-1) \cdot 4^{k-1}}
$$

We have already manipulated the negative of the upper bound. Thus using the corresponding hypergeometric anti-difference one writes

$$
\begin{aligned}
\frac{1}{2} \sum_{k=n+1}^{\infty} \frac{\left(\begin{array}{c}
2 \cdot k \\
k
\end{array}\right)}{(2 \cdot k-1) \cdot 4^{k-1}} & =\lim _{N \rightarrow \infty} s_{n+1}-s_{N} \\
& =\lim _{N \rightarrow \infty} \frac{(n+1) \cdot\left(\begin{array}{c}
2 \cdot(n+1) \\
n+1
\end{array}\right)}{(2 \cdot n+1) \cdot 4^{n}}-\frac{N \cdot\left(\begin{array}{c}
2 \cdot N \\
N
\end{array}\right)}{(2 \cdot N-1) \cdot 4^{N-1}} \\
& =\frac{(n+1) \cdot\left(\begin{array}{c}
2 \cdot(n+1) \\
n+1
\end{array}\right)}{(2 \cdot n+1) \cdot 4^{n}},
\end{aligned}
$$

which is the expected upper bound. For the lower bound we apply the same process. We have

$$
\frac{\left(\begin{array}{c}
2 \cdot k \\
k
\end{array}\right)}{2 \cdot(2 \cdot k+2) \cdot 4^{k-1}}=-\frac{\left(\begin{array}{c}
2 \cdot(k+1) \\
k+1
\end{array}\right)}{2 \cdot 4^{k}}+\frac{\left(\begin{array}{c}
2 \cdot k \\
k
\end{array}\right)}{2 \cdot 4^{k-1}}
$$

and therefore by telescoping we find

$$
\begin{aligned}
\frac{1}{2} \sum_{k=n+1}^{\infty} \frac{\left(\begin{array}{c}
2 \cdot k \\
k
\end{array}\right)}{(2 \cdot k+2) \cdot 4^{k-1}} & =\lim _{N \rightarrow \infty}-\frac{\left(\begin{array}{c}
2 \cdot N \\
N
\end{array}\right)}{2 \cdot 4^{N}}+\frac{\left(\begin{array}{c}
2 \cdot(n+1) \\
n+1
\end{array}\right)}{2 \cdot 4^{n}} \\
& =\frac{\left(\begin{array}{c}
2 \cdot(n+1) \\
n+1
\end{array}\right)}{2 \cdot 4^{n}}
\end{aligned}
$$

which is the expected lower bound.

Observe how close the lower and the upper bounds of (11) are. This could be interpreted from the following corollary. 


\section{Corollary 1.1.}

$$
\lim _{n \rightarrow \infty} \frac{2 \cdot \pi-\sum_{k=0}^{n} \frac{\left(\begin{array}{c}
2 \cdot k \\
k
\end{array}\right)}{(2 \cdot k+1) \cdot 4^{k-1}}}{\frac{\left(\begin{array}{c}
2 \cdot(n+1) \\
n+1
\end{array}\right)}{4^{n}}}=1
$$

Proof. This is straightforward from Theorem 1. Indeed, by dividing the member of the inequality (10) by $\frac{\left(\begin{array}{c}2 \cdot(n+1) \\ n+1\end{array}\right)}{2 \cdot 4^{n}}>0$ it follows that

$$
1 \leqslant \frac{\pi-\frac{1}{2} \sum_{k=0}^{n} \frac{\left(\begin{array}{c}
2 \cdot k \\
k
\end{array}\right)}{(2 \cdot k+1) \cdot 4^{k-1}}}{\frac{\left(\begin{array}{c}
2 \cdot(n+1) \\
n+1
\end{array}\right)}{2 \cdot 4^{n}}} \leqslant \frac{2 \cdot(n+1)}{2 \cdot n+1}
$$

Since

$$
\lim _{n \rightarrow \infty} \frac{2 \cdot(n+1)}{2 \cdot n+1}=1 \text {, }
$$

the expected result holds by application of the sandwich theorem.

In words we say that all the members of the inequalities in (11) are equivalent, that is, the limit of the ratio of any two among them is 1 . This corollary also tells us that the partial sum

$$
S_{n}^{(\pi)}:=\frac{1}{2} \sum_{k=0}^{n} \frac{\left(\begin{array}{c}
2 \cdot k \\
k
\end{array}\right)}{(2 \cdot k+1) \cdot 4^{k-1}}
$$

approaches $\pi$ as

$$
T_{n}:=\frac{\left(\begin{array}{c}
2 \cdot(n+1) \\
n+1
\end{array}\right)}{2 \cdot 4^{n}}
$$

approaches zero. It is observable that for a fixed positive integer $n$, the number of zeros right after the decimal point of the value of $T_{n}$ is close to the number of right decimal places of $\pi$ computed by $S_{n}^{(\pi)}$. The table of numerical values below illustrates this consequence.

Table 1: A slow convergence to $\pi$

\begin{tabular}{|l|l|l|l|l|l|}
\hline$n$ & 100 & 500 & 1000 & 10000 & 50000 \\
\hline$T_{n}$ & $0.1121 \ldots$ & $0.0503 \ldots$ & $0.0356 \ldots$ & $0.0112 \ldots$ & $0.0050 \ldots$ \\
\hline$S_{n}^{(\pi)}$ & $3.0292 \ldots$ & $3.0911 \ldots$ & $3.1059 \ldots$ & $3.1303 \ldots$ & $3.1365 \ldots$ \\
\hline \multicolumn{5}{|c|}{$\pi=3.1415 \ldots$} \\
\hline
\end{tabular}

As one can see, the infinite series (10) converges spectacularly slowly to $\pi$. The slow converging Leibniz-Gregory series representing the power series of the inverse tangent function evaluated at 1 converges even faster than (10). Nevertheless, we are not concerned with the speed of convergence. In the next section, we will use the inequalities in (11) as the centerpiece of an algorithmic approach to computing rational approximations to $\pi$ and $\pi+e$. 


\section{Algorithmic computation of rational approximations of $\pi$ and $\pi+e$}

We would like to start by mentioning that despite the word "approximation" which may suggest an inaccuracy in the computations, there is no such thing when dealing with symbolic manipulations. Depending on the available algorithms that wisely use the human techniques, the result of an algorithm based on symbolic computations gives exactly what could be handled by hand but in a very efficient way. In the present case, the manipulations are rather very simple and therefore, we can exactly produce the desired results. A simple Maxima (see Maxima's website (2020)) package with implementations of the algorithms of this section can be downloaded from the linkhttps://github.com/T3gu1a/PiplusErationals/blob/master/ Pipluserationals.mac.

Irrationality proofs of famous irrational numbers generally give a way to compute their rational approximations. Done by contradiction, these proofs use the fact that there is no integer in a unit open interval with integer bounds to show that the numerator or the denominator of a fraction representing the corresponding numbers cannot exist. It is motivated by this strategy that we do some observations to know what could be the outcome if $\pi$ is replaced by a rational number in (11).

First of all, we need to find close integers to $\pi$ among which its integer part could belong to. Although this is well known from mathematical books, we avoid such prior knowledge so that the approach of this paper remains independent and selfexplanatory. The infinite series (9) has a decreasing partial sum which starts with the value 4 . Therefore $\pi \leqslant 4$. On the other hand, the infinite series (10) has an increasing partial sum that reaches values greater than 3 after its hundredth term as presented in Table 1 . Thus $3 \leqslant \pi \leqslant 4$ which implies that 3 can be considered as a very close integer to $\pi 3$

Now let $\frac{p+1}{q}>3$ for positive integers $p, q$, be a rational number that can replace $\pi$ in (11). Substituting $\pi$ by $(p+1) / q$ and $n$ by $p$ in (11) yields

$$
\frac{\left(\begin{array}{c}
2 \cdot(p+1) \\
p+1
\end{array}\right)}{2 \cdot 4^{p}} \leqslant \frac{p+1}{q}-\frac{1}{2} \sum_{k=0}^{p} \frac{\left(\begin{array}{c}
2 \cdot k \\
k
\end{array}\right)}{(2 \cdot k+1) \cdot 4^{k-1}} \leqslant \frac{(p+1) \cdot\left(\begin{array}{c}
2 \cdot(p+1) \\
p+1
\end{array}\right)}{(2 \cdot p+1) \cdot 4^{p}}
$$

which after division by $p+1$ leads to

$$
\frac{\left(\begin{array}{c}
2 \cdot(p+1) \\
p+1
\end{array}\right)}{2 \cdot(p+1) \cdot 4^{p}} \leqslant \frac{1}{q}-\frac{1}{2 \cdot(p+1)} \sum_{k=0}^{p} \frac{\left(\begin{array}{c}
2 \cdot k \\
k
\end{array}\right)}{(2 \cdot k+1) \cdot 4^{k-1}} \leqslant \frac{\left(\begin{array}{c}
2 \cdot(p+1) \\
p+1
\end{array}\right)}{(2 \cdot p+1) \cdot 4^{p}} .
$$

These last inequalities indicate quite well the precision of the fractional part of $(p+$ $1) / p$. For all integers $n$, let

$$
L_{n}^{(\pi)}:=\frac{\left(\begin{array}{c}
2 \cdot(n+1) \\
n+1
\end{array}\right)}{2 \cdot(n+1) \cdot 4^{n}}
$$

${ }^{3} \mathrm{By}$ geometric observation, $\pi$ (i.e the area of the unit circle) is strictly less than the area of the square of side's length 2 which allows deducing that the integer part of $\pi$ is 3 . 
and

$$
U_{n}^{(\pi)}:=\frac{\left(\begin{array}{c}
2 \cdot(n+1) \\
p+1
\end{array}\right)}{(2 \cdot n+1) \cdot 4^{n}} .
$$

The following algorithm computes the possible values of $(p+1) / q$ for a given range of integers for $p$.

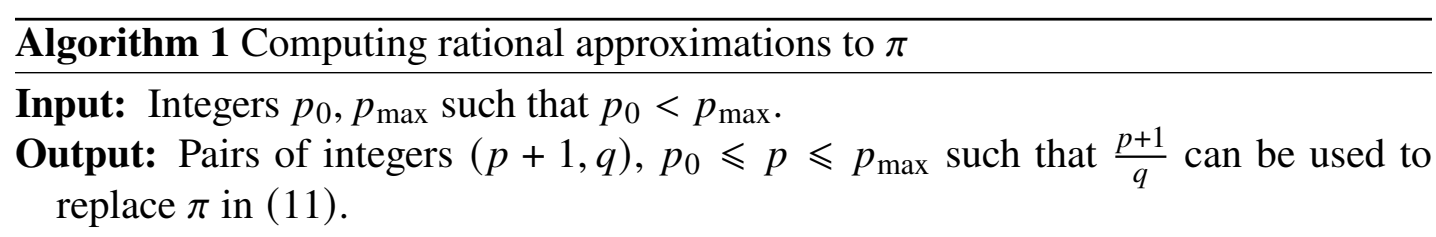

1. Set $L:=\{\}$ and

$$
E(m, n):=\frac{1}{m}-\frac{S_{n}^{(\pi)}}{n+1}
$$

where $S_{n}^{(\pi)}$ is given by $(18)$.

2. For $\max \left\{p_{0}, 6\right\} \leqslant p \leqslant p_{\max }$ do

(a) $q=\left\lfloor\frac{p}{3}\right\rfloor$

(b) While $E(q, p) \leqslant U_{p}^{(\pi)}$ do

i. If $E(q, p) \geqslant L_{p}^{(\pi)}$ then add $(p+1, q)$ to $L$.

ii. $q=q-1$

3. Return $L$.

This algorithm is easily justified from our previous development and Section 2 . The expression (22) represents the middle term of the inequalities given in (19). Since for a fixed value of $n, E(m, n)$ increases as $m$ decreases, the loops used in the algorithm are properly deduced. The loop in step 2 starts at least at 6 because values for the denominator $q$ must be at least 2 since $\pi$ is clearly not an integer. The step $2 a$ comes from the fact that 3 is a close integer to $\pi(\pi \geqslant 3)$.

When running our Maxima implementation for input integers less than 10000, after several hours of computations we get the following list of integer pairs

$[22,7],[44,14],[355,113],[710,226],[1065,339],[1420,452],[1775,565]$,

[2130, 678], [2485, 791], [2840, 904], [3195, 1017], [3550, 1130], [3905, 1243], [4260, 1356], [4615, 1469], [4970, 1582], [5325, 1695], [5680, 1808], [6035, 1921], [6390, 2034], [6745, 2147], [7100, 2260], [7455, 2373], [7810, 2486],

[8165, 2599], [8520, 2712], [8875, 2825], [9230, 2938], [9585, 3051], [9940, 3164] 
whose ratios correspond to only two rational numbers, namely,

$$
\frac{22}{7}, \text { and } \frac{355}{113}
$$

with, respectively, 2 and 28 occurrences. This can be seen as records: 22/7 remains a record until the denominator has three digits, and 355/113 remains unbeaten for denominators less than 4000 . One needs a very good computer to continue these observations. will the record of 355/113 be broken at some point? We could propose an answer to this question from the known part of the regular continue fraction of $\pi$. (Eymard and Lafon, 1999, page 73) presents an evaluation of the error given by the first convergents of the continue fraction of $\pi$ and, it shows that the record of 355/113 could be broken by the convergent $52163 / 16604$. However, this is not so sure because the computations given in that book shows that the record of $22 / 7$ is broken when the denominator reaches 57 which is not the case in our approach. Therefore our algorithm clearly filters the set of convergents of $\pi$.

For the case of $\pi+e$ some little modifications need to be added to Algorithm 1. First we reestablish a well known inequality for

$$
e-\sum_{k=0}^{n} \frac{1}{k !}=\sum_{k=n+1}^{\infty} \frac{1}{k !}
$$

for all positive integers $n$, where $e$ is taken as $\sum_{n=0}^{\infty} \frac{1}{n !}$. We have

$$
\begin{aligned}
\sum_{k=n+1}^{\infty} \frac{1}{k !} & =\frac{1}{(n+1) !}+\frac{1}{(n+1) ! \cdot(n+2)}+\frac{1}{(n+1) ! \cdot(n+2) \cdot(n+3)}+\cdots \\
& =\frac{1}{(n+1) !}\left(1+\frac{1}{n+2}+\frac{1}{(n+2) \cdot(n+3)}+\frac{1}{(n+2) \cdot(n+3) \cdot(n+4)}+\cdots\right) \\
& \leqslant \frac{1}{(n+1) !} \cdot \lim _{N \rightarrow \infty}\left(1+\frac{1}{n+1}+\frac{1}{(n+1)^{2}}+\frac{1}{(n+1)^{3}}+\cdots+\frac{1}{(n+1)^{N}}\right) \\
& =\frac{1}{(n+1) !} \cdot \lim _{N \rightarrow \infty} \frac{1-\frac{1}{(n+1)^{N+1}}}{1-\frac{1}{n+1}}, \text { summation of a geometric series } \\
& =\frac{1}{n \cdot n !} .
\end{aligned}
$$

Therefore we get

$$
0 \leqslant e-\sum_{k=0}^{n} \frac{1}{k !} \leqslant \frac{1}{n \cdot n !} .
$$

Summing up the inequalities (11) and 25 member to member gives

$$
\frac{\left(\begin{array}{c}
2 \cdot(n+1) \\
n+1
\end{array}\right)}{2 \cdot 4^{n}} \leqslant \pi+e-\frac{1}{2} \sum_{k=0}^{n} \frac{\left(\begin{array}{c}
2 \cdot k \\
k
\end{array}\right)}{(2 \cdot k+1) \cdot 4^{k-1}}-\sum_{k=0}^{n} \frac{1}{k !} \leqslant \frac{(n+1) \cdot\left(\begin{array}{c}
2 \cdot(n+1) \\
n+1
\end{array}\right)}{(2 \cdot n+1) \cdot 4^{n}}+\frac{1}{n \cdot n !} .
$$


Thus to compute rational approximations $\frac{p+1}{q}$ to $\pi+e$ we have to consider the following inequalities

$$
\frac{\left(\begin{array}{c}
2 \cdot(p+1) \\
p+1
\end{array}\right)}{2 \cdot(p+1) \cdot 4^{p}} \leqslant \frac{1}{q}-\frac{1}{p+1}\left(\sum_{k=0}^{p} \frac{\left(\begin{array}{c}
2 \cdot k \\
k
\end{array}\right)}{2 \cdot(2 \cdot k+1) \cdot 4^{k-1}}+\frac{1}{k !}\right) \leqslant \frac{\left(\begin{array}{c}
2 \cdot(p+1) \\
p+1
\end{array}\right)}{(2 \cdot p+1) \cdot 4^{p}}+\frac{1}{p \cdot(p+1) !} .
$$

Next, as previously we find close integers to

$$
\pi+e=\sum_{n=0}^{\infty} \frac{\left(\begin{array}{c}
2 \cdot n \\
n
\end{array}\right)}{2 \cdot(2 \cdot n+1) \cdot 4^{n-1}}+\frac{1}{n !} .
$$

The infinite series in 28 has an increasing partial sum since its general term is positive and moreover, for $n \geqslant 100$ the values taken by this partial sum are greater than 5. Therefore $\pi+e \geqslant 5$. On the other hand, we have

$$
\begin{aligned}
e & =\sum_{n=0}^{\infty} \frac{1}{n !} \\
& =1+1+\frac{1}{2}+\frac{1}{3 \cdot 2}+\frac{1}{4 \cdot 3 \cdot 2}+\cdots \\
& \leqslant 1+\sum_{n=0}^{\infty} \frac{1}{2^{n}} \\
& =1+\frac{1}{1-\frac{1}{2}}=3
\end{aligned}
$$

which implies that $\pi+e \leqslant 7$ since we have already shown that $\pi \leqslant 4$. Thus our close integers to $\pi+e$ are 5 and 64 . Let

$S_{n}^{(\pi+e)}:=\sum_{k=0}^{n} \frac{\left(\begin{array}{c}2 \cdot k \\ k\end{array}\right)}{2 \cdot(2 \cdot k+1) \cdot 4^{k-1}}+\frac{1}{k !}, L_{n}^{(\pi+e)}:=L_{n}^{(\pi)}, U_{n}^{(\pi+e)}:=\frac{\left(\begin{array}{c}2 \cdot(n+1) \\ n+1\end{array}\right)}{(2 \cdot n+1) \cdot 4^{n}}+\frac{1}{n \cdot(n+1) !}$.

Our algorithm to compute rational approximations to $\pi+e$ goes as follows.

As one can see, the algorithms 1 and 2 are similar and therefore similarly justified. Running Algorithm 2 for input numerators of rational approximations to $\pi+e$ less than 10000 yields

$$
[[920,157],[1840,314]]
$$

for input $d=5$, and<smiles>c1ccccc#1</smiles>

for input $d=6$. From these computations it is natural to think that the integer part of $\pi+e$ is 5 . The integer pairs in (31) correspond to the same rational number

$$
\frac{920}{157}
$$

${ }^{4}$ Further manipulations may leads to the fact that 5 is the necessary integer part but we choose to leave the algorithm decide (see (31) and (32) 


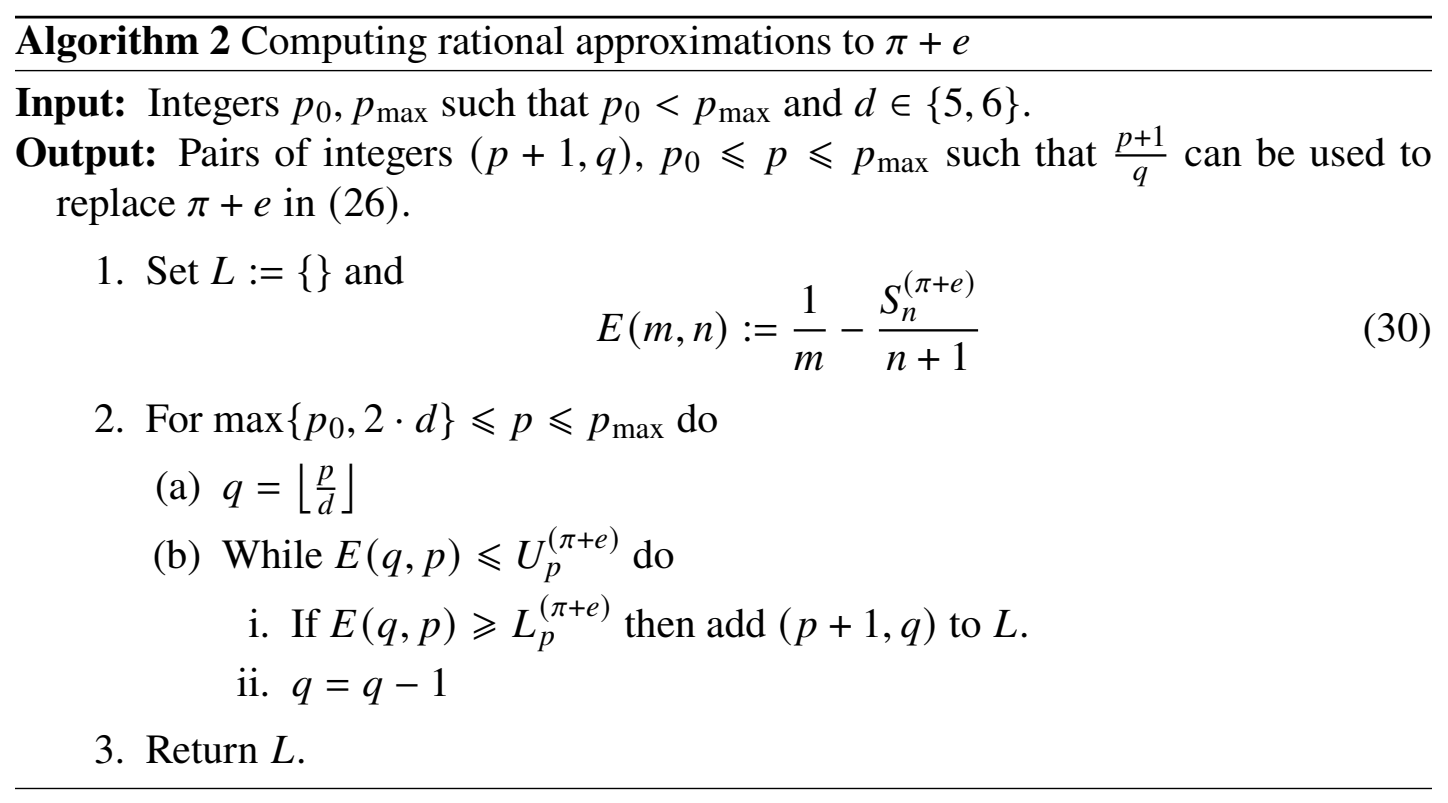

which is the only record for rational approximations to $\pi+e$ with denominator less than 2000. It is thanks to such an observation that we say that $920 / 157$ is the best rational approximation to $\pi+e$ with a denominator of at most three digits. As previously said in the case of $\pi$, a quite powerful computer can help to continue the observations and see whether this record is broken for larger denominators. Nevertheless, one can consider $920 / 157$ as a special number for $\pi+e$, at least as Yuelü and Milü are for $\pi$. The most important fact, however, is the filtration of convergents that could lead to finitely many rational approximations unlike the regular continued fraction approach.

\section{Discussions}

What about $\pi-e$ ? Well, it is easy to deduce that $0 \leqslant \pi-e \leqslant 1$ from our development. This reveals that the denominators to be sought are larger than their corresponding numerators. Our method could probably be adapted to this case but if we look in particular at the division by integers close to $\pi-e$ (see step 2 in algorithm 2 ), then we observe an impossible operation. Even if we manage to adapt algorithm 2, the approximation quality may be poorer.

Do we have an idea of the structure of $\pi+e$ ? The obtained 22/7 and 355/113 from the use of algorithm 1 are convergents of the continued fraction of $\pi$. Let us see whether 920/157 represent a convergent of the continued fraction of $\pi+e$. Note that the regular continued fraction of a rational number has two equivalent representations written as

$$
\left[c_{0} ; c_{1}, \ldots, c_{n}\right]=\left[c_{0} ; c_{1}, \ldots,\left(c_{n}-1\right), 1\right]
$$


for an integer $c_{0}$ and positive integers $c_{1}, \ldots, c_{n}$. For $920 / 157$ one gets

$$
\frac{920}{157}=[5 ; 1,6,7,3]=[5 ; 1,6,7,2,1]=5+\frac{1}{1+\frac{1}{6+\frac{1}{7+\frac{1}{2+\frac{1}{1}}}}} .
$$

One can prove that the continued fraction representation (34) is a convergent of any real number strictly between

$$
\left[c_{0} ; c_{1}, \ldots, c_{n}-1,2\right] \text { and }\left[c_{0} ; c_{1}, \ldots, c_{n}+1\right] .
$$

In the case of $920 / 157$ that is

$$
[5 ; 1,6,7,2,2]=\frac{1547}{264} \approx 5.8598484 \text { and }[5 ; 1,6,7,4]=\frac{1213}{207} \approx 5.8599033 \text {. }
$$

Therefore if we can trust the first digits of the numerical approximation of $\pi+e \approx$ 5.8598744820488385 given by all the systems at the present time, then it is clear that $920 / 157$ is a convergent of $\pi+e$, that is to say that the regular continued fraction of $\pi+e$ starts as

$$
\pi+e=[5 ; 1,6,7,3, \ldots]
$$

Using Beukers (2000) definition of the rational approximation quality, the one of $920 / 157$ is about

$$
-\frac{\log \left(\pi+e-\frac{920}{157}\right)}{\log (157)} \approx 2.608508457338876
$$

which is interesting and may encourage further investigations in our approach for a rationality or an irrationality proof of $\pi+e$ still unsolved.

On the other hand, one can combine decimal approximations and regular continued fraction representation to obtain rational approximations to $\pi+e$. For example, using the rational number $x:=5.859874482048838$, by application of an algorithm that computes the regular continued fraction representation, we find

$$
x=[5 ; 1,6,7,3,21,2,1,2,2,1,1,2,3,3,2],
$$

which starts as $(38)$. Note that the appearance of 21 shows the quality of the approximation given by $[5 ; 1,6,7,3]$. Indeed, it is easy to see that small numbers in the regular continued fraction lead to a less good quality of a convergent than large numbers. In

\footnotetext{
${ }^{5}$ The numerical value given for $\pi+e$ is the result of an addition. Although the decimal places given for $\pi$ and $e$ are correct we cannot be completely sure about $\pi+e$. Nevertheless there may be no error for the first billion decimal places.
} 
the case of $\pi+e$, one has to use 50 decimal places to see a number greater than 21 appearing in its regular continued fraction. The corresponding rational is

$$
\frac{7992197684740010333102543}{1363885473865240420705943}
$$

which has a denominator with 25 digits, and this is more likely the next record after $920 / 157$ !

What else? In the sense of our development one can see 920/157 as the best rational number to substitute $\pi+e$ when the latter appears in calculations. The use of such an approximation is generally better than an approximated numerical value because it minimizes the error in a better way. For example, to substitute $1 /(\pi+e)$ one only needs to use $157 / 920$ which is obtained without another approximation. However, as we pointed out earlier, the most important fact in our approach is that it can lead to a finite number of good rational approximations extracted from the regular continued fraction of $\pi$ or $\pi+e$. In the case of $\pi$, this could lead to a new bound of its irrationality measure. And for $\pi+e$ this could be seen as an important exploratory computation to know its structure.

Nevertheless, it should be mentioned that the interest in studying the number $\pi+e$ has no justification in applications yet. We do not yet see its appearance in applied sciences as the case of $\pi$ or $e$. Knowing the addition of these two numbers reveals a characteristic of the method that nature uses to add. It is always fascinating to see how close rational numbers can come. Just as Laplace was surprised when he saw the connection between the circle (i.e $\pi$ ) and the game of heads or tails through Wallis' formula Eymard and Lafon (1999), we wonder well but being less skeptical on what could be the result of 'adding' a circle and a proportional growing (i.e $\pi+e$ ).

\section{References}

Agarwal, R., Agarwal, H., and Sen, S. (2013). Birth, growth and computation of pi to ten trillion digits. Adv. Differ. Equ., 2013:100.

Bailey, D., Borwein, P., and Plouffe, S. (1997). On the rapid computation of various polylogarithmic constants. Math. Comp., 66(218):903-913.

Beukers, F. (2000). A rational approach to pi. Nieuw archief voor wiskunde. Serie 5, 1(4):372-379.

Eymard, P. and Lafon, J.-P. (1999). Autour du Nombre $\pi$, Volume 1443. Hermann, 75015 Paris.

Gosper, B. (2015). Continued fraction arithmetic. https://perl.plover.com/ classes/cftalk/INF0/gosper.txt 
Gosper, R. W. (1978). Decision procedure for indefinite hypergeometric summation. Proceedings of the National Academy of Sciences, 75(1):40-42.

Maxima's website (2020). Maxima, A Computer Algebra System. http://maxima . sourceforge.net/.

Mullican, T. (2020). Calculating Pi: My attempt at breaking the pi World Record. https://blog.timothymullican.com/ calculating-pi-my-attempt-breaking-pi-record.

Pettofrezzo, A. J. and Byrkit, D. R. (1970). Elements of Number Theory. Prentice Hall, Englewood Cliffs.

Teguia T., B. (2020). Power Series Representations of Hypergeometric Types and Non-Holonomic Functions in Computer Algebra. PhD thesis, University of Kassel.

Zeilberger, D. and Zudilin, W. (2020). The irrationality measure of pi is at most 7.103205334137... arXiv preprint arXiv:1912.06345v2. 